\title{
Conceitos $\epsilon$ aspectos da abordagem com base no desempenho na avaliação da durabilidade de estruturas de concreto armado expostas a ambientes agressivos

\begin{tabular}{c}
\hline GUSTAVO BOSEL WALLY - DoutORANDO \\
\hline orcid.org/0000-0003-3008-3815 \\
UniverSIDADE FEDERAL do Rio Grande DO SUL
\end{tabular} PPGEC-UFRGS

\author{
FÁBIO COSTA MAGALHÃES - ProfESSOR DoutoR \\ orcid.org/0000-0001-8463-1501 \\ Instituto Federal do Rio Grande do Sul, Campus Rio Grande \\ LEMCC-IFRS
}

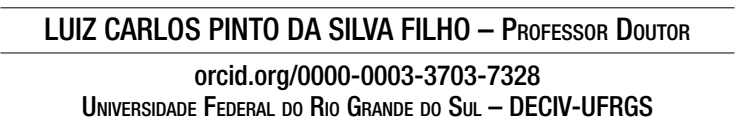

\section{RESUMO}

A DURABILIDADE DAS ESTRUTURAS DE CONCRETO ARMADO É UMA PREOCUPAÇÃO GENERALIZADA ENTRE OS ENVOLVIDOS COM A CONSTRUÇÃO CIVIL. EM MUITOS CASOS, AS PROPRIEDADES DO CONCRETO NÃO ATENDEM A REQUISITOS MÍNIMOS, REDUZINDO A VIDA ÚTIL DAS ESTRUTURAS, PRINCIPALMENTE DEVIDO À CORROSÃO DAS ARMADURAS DESENCADEADA PELA PENETRAÇÃO DE AGENTES AGRESSIVOS, COMO OS ÍONS CLORETO E O DIÓXIDO DE CARBONO. A UTILIZAÇÃO DA ABORDAGEM COM BASE NO DESEMPENHO E DOS INDICADORES DE DURABILIDADE DO CONCRETO, PORTANTO, TEM SIDO DISCUTIDA MUNDIALMENTE COMO METODOLOGIA DE AVALIAÇÃO

DAS CARACTERÍSTICAS DO CONCRETO E DE SEU POTENCIAL DE DURABILIDADE FRENTE A DETERMINADO AMBIENTE. ISSO OCORRE PORQUE CONCRETOS QUE SEGUEM OS MESMOS PARÂMETROS PRESCRITIVOS PODEM APRESENTAR RESULTADOS DIFERENTES QUANDO AVALIADAS SUAS PROPRIEDADES RELACIONADAS À DURABILIDADE. ESTE TRABALHO DISCUTE ASPECTOS DA ABORDAGEM COM BASE NO DESEMPENHO, APRESENTANDO RESULTADOS EXPERIMENTAIS E AS PROPOSTAS ADOTADAS POR DIFERENTES PAÍSES QUE IMPLEMENTARAM ESSA ABORDAGEM EM SUAS METODOLOGIAS DE PROJETO DE DURABILIDADE DE ESTRUTURAS DE CONCRETO ARMADO.

Palavras-chave: durabilidade do concreto, abordagem com base no desempenho, indicadores de durabilidade.

\section{INTRODUÇÃO}

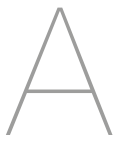

tualmente, a durabilidade das estruturas de concreto armado é uma grande preocupação por motivos de segurança e economia. A principal causa da deterioração precoce deste tipo de estruturas é a corrosão das armaduras, causada pela penetração de cloretos ou de dióxido de carbono. Assim, esforços têm sido somados na busca por métodos de ensaios acelerados que quantifiquem a resistência do concreto à deterioração, no desenvolvimento de modelos de previsão de vida útil confiáveis e na realização de projetos de durabilidade, a fim de conferir maior vida útil e menor custo de ciclo de vida às estruturas, além de propiciar um correto planejamento das intervenções de manutenção (BAROGHEL-BOUNY, NGUYEN e DANGLA, 2009; BEUSHAUSEN, TORRENT $\mathrm{C}$ ALEXANDER, 2019).

A abordagem prescritiva tradicionalmente adotada nas considerações sobre a durabilidade de normas que 
versam sobre o projeto de estruturas de concreto armado, como, por exemplo, a ABNT NBR 6118:2014 e a EN 206, limita-se a estipular valores-limite para parâmetros como consumo de cimento mínimo, $f_{c k}$ mínimo e relação água/aglomerante máxima, no caso das estruturas correntes. Esses limites, embora tendam a afetar algumas propriedades relacionadas à durabilidade do concreto, não estabelecem condições definitivas para que o concreto seja realmente durável no ambiente ao qual será exposto. No caso de estruturas especiais, a ABNT NBR 6118:2014, em seu item 7.4.2, apresenta a ideia da utilização de indicadores de durabilidade como metodologia de avaliação do desempenho da estrutura quanto à durabilidade frente à classe de agressividade prevista em projeto, sem citar, no entanto, nenhum mecanismo de ensaio, tampouco valores de referência para a validação dos resultados.

Torrent et al. (2016) destacam ainda que dentro da metodologia prescritiva pode haver variações significativas nos valores-limite indicados para uma mesma classe de agressividade. Por exemplo, países que adotaram a EN 206, como, entre outros, Dinamarca, Portugal e Reino Unido, modificaram significativamente as recomendações acerca da relação água/aglomerante e do consumo de cimento mínimo. Assim, tomando como exemplo a classe XS3 ${ }^{1}$ e considerando os parâmetros adotados nos diversos países, observa-se que os limites para a relação água/aglomerante podem variar de 0,35 a 0,50, enquanto o consumo de cimento mínimo apresenta valores entre 150 e $400 \mathrm{~kg} / \mathrm{m}^{3}$.
Neste cenário, é crescente a demanda pela inclusão de conceitos e métodos avançados de controle de qualidade do concreto e de previsão de vida útil das estruturas, utilizando mecanismos que realmente avaliem as propriedades relacionadas à durabilidade do concreto, especialmente em relação à ocorrência da corrosão das armaduras (BAROGHEL-BOUNY et al., 2011). Assim, a filosofia e a implementação da abordagem com base no desempenho no projeto de durabilidade e no controle de qualidade das estruturas executadas têm sido fomentadas, principalmente no campo das pesquisas, visando preencher as lacunas apresentadas pela metodologia prescritiva e contribuir para a concepção de estruturas de concreto armado mais duráveis.

\section{PRINCÍPIOS DA ABORDAGEM COM BASE NO DESEMPENHO NA AVALIAÇÃO DA DURABILIDADE DE ESTRUTURAS DE CONCRETO ARMADO}

$\mathrm{Na}$ abordagem com base no desempenho, diferentemente do que ocorre na abordagem prescritiva, o potencial de uma determinada estrutura em cumprir com sua vida útil de projeto é avaliado considerando basicamente a capacidade do concreto de resistir à deterioração, a ser mensurada através de um ou mais indicadores de durabilidade. A consideração do potencial de durabilidade da estrutura com base no seu desempenho é de fundamental importância, visto que concretos que obedecem aos mesmos parâmetros prescritivos podem apresentar diferentes comportamentos quando avaliadas suas propriedades relacionadas à durabilidade.

A Tabela 1 e a Figura 1 apresentam alguns dos resultados obtidos por Wally (2019). No estudo, foram avaliados sete diferentes concretos, sendo um de referência ( $R$ 45), com consumo de cimento de $400 \mathrm{~kg} / \mathrm{m}^{3}$, três com substituição parcial do cimento por sílica ativa nos teores de 5, 10 e $20 \%$ (SF 5, SF 10 e SF 20, respectivamente) e outros três com substituição parcial de cimento por metacaulim, também nos teores de 5, 10 e 20 \% (MK 5, $\checkmark$ Tabela 1 - Influência do tipo e teor de adições minerais no desempenho de concretos frente à penetração de cloretos

\begin{tabular}{|cccc|}
\hline Concreto & $f_{\text {om }}(\mathrm{M}$ Pa $)$ & $D_{\text {nssm }}\left(\mathbf{1 0}^{-12} \mathrm{~m}^{2} / \mathrm{s}\right)^{*}$ & $\mathrm{P}_{f}(\%)^{* *}$ \\
\hline R 45 & 63,22 & 2,84 & 49,51 \\
\hline SF 5 & 63,46 & 1,40 & 1,86 \\
\hline SF 10 & 70,88 & 0,90 & 0,19 \\
\hline SF 20 & 74,51 & 0,27 & 0,00 \\
\hline MK 5 & 62,86 & 2,14 & 15,10 \\
MK 10 & 63,55 & 0,96 & 0,52 \\
MK 20 & 66,99 & 0,62 & 0,03 \\
* Coeficiente de difusão determinado utilizando o método normatizado pela NT Build 492:1999; \\
** Probabilidade de corrosão à idade de 50 anos, considerando cobrimento nominal de 50 mm, calculada com base no modelo \\
apresentado por Giørv (2015).
\end{tabular}


MK 10 e MK 20, respectivamente). Em todos os concretos foi adotada relação água/aglomerante igual a 0,45.

Com base nos dados apresentados na Tabela 1, destaca-se que os concretos R 45, SF 5, MK 5 e MK 10 apresentam variações de resistência à compressão média inferiores a $1 \mathrm{MPa}$. Essa diferença é bastante pequena quando avaliado o grau de precisão do ensaio em questão, podendo a variação da resistência de corpos de prova de um mesmo traço ser superior a esse valor. No entanto, ao avaliar os coeficientes de difusão $\left(D_{\text {nssm }}\right)$, nota-se que, dentre os mesmos quatro concretos, a difusividade pode ser reduzida em até $66,2 \%$. Ademais, como se vê na Figura 1, não há correlação entre a resistência à compressão e o coeficiente de difusão, principalmente quando utilizadas adições minerais. Dessa forma, ainda que alguns concretos apresentem altas resistências à compressão, sua difusividade pode não ser suficientemente baixa para que a estrutura cumpra com sua vida útil de projeto frente ao ambiente ao qual será exposta.

A consideração dos indicadores de durabilidade, por sua vez, possibilita a

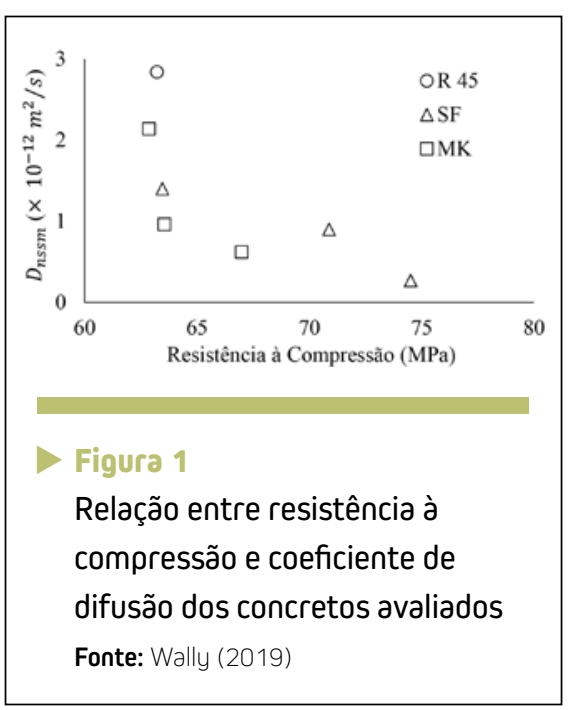

análise direta da influência de diversos componentes do concreto na durabilidade da estrutura. $\mathrm{O}$ emprego de materiais cimentícios suplementares ou de aditivos químicos que visam aumentar a durabilidade, ainda que afete sutilmente aspectos como consumo de cimento e resistência à compressão, pode resultar em um concreto significativamente mais durável, se esses materiais forem selecionados, dosados e empregados corretamente. Assim, ao propor a avaliação das características do produto final, ao invés de somente estipular parâmetros a serem seguidos, a abordagem com base no desempenho contribui para o aumento da liberdade dos responsáveis pela dosagem do concreto e pelo projeto de durabilidade, possibilitando o uso de misturas de concreto inovadoras e potencialmente contribuindo para a redução dos danos ambientais, caso dos concretos com baixo consumo de cimento e/ou com utilização de altos teores de adições minerais.

Salienta-se, porém, que apesar de abranger a utilização de indicadores de durabilidade, a abordagem com base no desempenho não se limita somente a eles, mas engloba também a consideração da agressividade do ambiente, a vida útil requerida e o plano de manutenções da estrutura. Os princípios básicos do projeto de durabilidade baseado em desempenho são apresentados na Figura 2.

Deve-se atentar, de acordo com Beushausen et al. (2016), para o fato de que o projeto baseado em desempenho pode requerer a inclusão de requisitos prescritivos para parâmetros dos materiais, como tipo de cimento, por exemplo, uma vez que a interpretação dos indicadores de durabilidade pode depender do tipo de material utilizado.
Por exemplo, concretos produzidos com diferentes aglomerantes podem apresentar valores semelhantes para resistividade elétrica, mas mecanismos de fixação de cloretos diferentes, proporcionando, assim, potenciais de durabilidade distintos se expostos a um ambiente rico em cloretos.

\section{INDICADORES DE DURABILIDADE}

Com vistas ao fato de que a durabilidade das estruturas de concreto armado está intimamente relacionada à espessura da camada de cobrimento e à capacidade desta de impedir a penetração de agentes agressivos, avaliar as características do concreto por meio de métodos confiáveis é de fundamental importância para uma previsão mais precisa da vida útil da estrutura. Assim, na abordagem com base no desempenho é desejável que se avalie ao menos um indicador de durabilidade que reflita as propriedades gerais de durabilidade do concreto quando em suas primeiras idades, de maneira análoga à resistência à compressão avaliada aos 28 dias, que é um índice simples e relativo que reflete as propriedades mecânicas gerais do concreto. Podem ser tomadas como exemplo a avaliação do coeficiente de difusão de cloretos, no caso de estruturas expostas a ambientes marinhos, e a determinação da permeabilidade ao ar, no caso de concretos sujeitos a ambientes urbanos ou industriais.

Destaca-se também que a utilização dos indicadores de durabilidade, além de possibilitar a análise do potencial de durabilidade do concreto através da moldagem de corpos de prova antes da execução da estrutura, permite a avaliação in loco do material 
executado. É necessário perceber, no entanto, que os valores obtidos para os indicadores de durabilidade em concretos moldados serão diferentes daqueles tomados em amostras extraídas da estrutura e, ainda, ambos serão diferentes da capacidade real do concreto executado de resistir à penetração de agentes nocivos, de modo similar aos distintos valores obtidos para resistência potencial $\left(f_{c}\right)$, resistência dos corpos de prova extraídos $\left(f_{c, \text { ext }}\right)$ e resistência efetiva $\left(f_{\mathrm{c}, \mathrm{ef}}\right)$, quando avaliada a resistência à compressão do concreto.

Como resultado de vários anos de pesquisas sobre a durabilidade do concreto, diversas metodologias de ensaio foram desenvolvidas visando mensurar as propriedades de transporte do concreto (TORRENT, 2018). Esses ensaios podem despender minutos, caso da avaliação da resistividade elétrica superficial, algumas horas, se empregados métodos baseados na migração iônica, ou até mesmo algumas semanas, caso de métodos de carbonatação acelerada ou difusão de cloretos.

Embora tendam a ser mais confiáveis do ponto de vista da simulação das condições reais de exposição do concreto a um ambiente agressivo, a elevada duração de alguns ensaios dificulta o uso de seus resultados como mecanismo de verificação da conformidade do concreto em relação aos aspectos de durabilidade. Dessa forma, métodos mais rápidos e que apresentem boa repetibilidade e boa correlação com situações de exposição real têm sido mais adotados como indicadores de durabilidade, caso, por exemplo, de metodologias de avaliação baseadas na migração iônica, na resistividade e na permeabilidade do concreto. Alexander e Thomas (2015) apontam

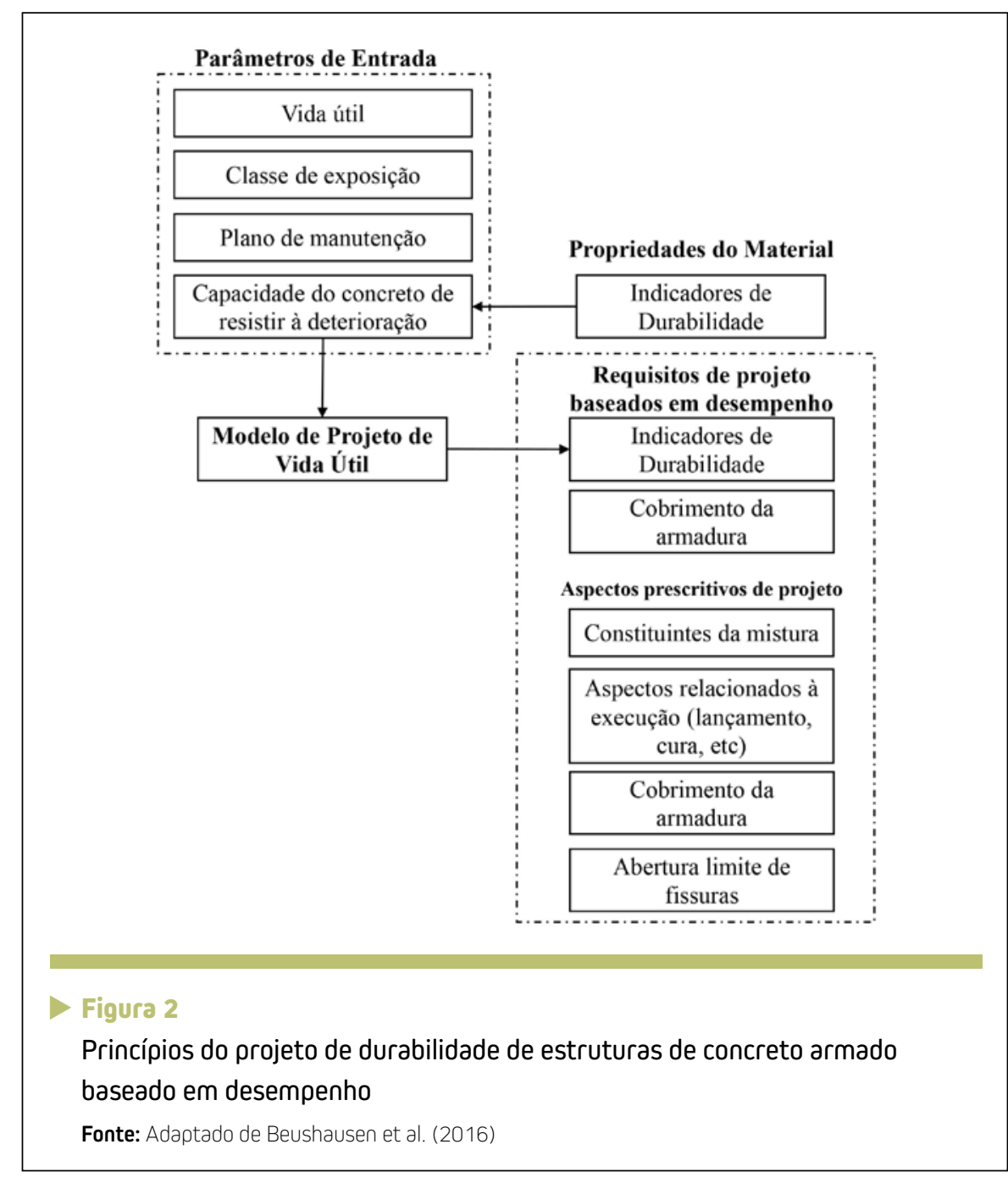

ainda que esses ensaios devem ser de fácil interpretação no contexto da engenharia, de simples mensuração e de resultados confiáveis, além de sensíveis a aspectos relacionados à dosagem do concreto e à execução da estrutura, como tipo de cimento, utilização de adições minerais, lançamento e cura.

$\mathrm{Na}$ implementação da abordagem com base no desempenho, destacam-se a África do Sul, a Suíça e alguns países nórdicos. A África do Sul desenvolveu um arcabouço de métodos que determinam o "índice de durabilidade" do concreto, considerando a resistência à penetração de cloretos, a permeabilidade ao oxigênio e a ab- sorção de água. Os resultados obtidos através desses ensaios são aplicados a modelos de previsão de vida útil, que podem variar a depender do ambiente ao qual o concreto será exposto - a saber, modelos baseados na penetração de cloretos ou de dióxido de carbono, por exemplo (TORRENT, 2018). Na Suiça, a norma SIA 262:2013 apresenta metodologias de testes de carbonatação acelerada, absorção de água e migração de cloretos, além de apresentar valores-limite e parâmetros para avaliação da conformidade do concreto. Por sua vez, países como Holanda, Noruega e Suécia adotam, principalmente em grandes obras de infraestrutura, o 
método de migração de cloretos normatizado pela NT Build 492:1999, associado a modelos probabilísticos de previsão de vida útil.

Como se vê, somente em alguns poucos países a utilização dos indicadores de durabilidade como forma de avaliação das características relacionadas à durabilidade do concreto e como parâmetro de entrada em modelos de previsão de vida útil está bem implementada. No geral, há a necessidade da normatização de metodologias confiáveis que forneçam aos engenheiros parâmetros de avaliação a serem seguidos. Além disso, para que exista a possibilidade da realização de projetos de durabilidade com base no desempenho, é necessária a consolidação de modelos preditivos de vida útil que descrevam de maneira fidedigna o processo de deterioração pelo qual a estrutura de concreto armado passará ao ser exposta a um ambiente agressivo.

\section{CONSIDERAÇÕES FINAIS}

Em nível mundial, a abordagem da durabilidade de estruturas de concreto armado caminha em direção às avaliações de desempenho. Assim, a introdução dos indicadores de durabilidade e de modelos de previsão de vida útil apresentam-se como aspectos fundamentais na avaliação do potencial de durabilidade de elementos estruturais sujeitos a ambientes agressivos.

Discutiu-se neste trabalho que concretos que possuem a mesma relação água/aglomerante e que pertencem à mesma classe de resistência podem apresentar comportamentos significativamente distintos quando avaliados sob a ótica da durabilidade em ambientes agressivos. Assim, mostra-se necessária a consolidação de normas técnicas acerca da durabilidade do concreto baseadas na avaliação de indicadores de durabilidade, em detrimento ao uso de métodos prescritivos. Além disso, os valores obtidos através dos indicadores de durabilidade podem ainda ser associados às análises numéricas de previsão de vida útil das estruturas, como já ocorre em alguns países nórdicos, a fim de contribuir para o dimensionamento de elementos estruturais capazes de cumprir com sua vida útil de projeto, garantindo o desempenho desejado e evitando custos prematuros com manutenções e reparos.

A filosofia da abordagem com base no desempenho mostra-se bem difundida em alguns países. No contexto brasileiro, porém, está majoritariamente restrita ao meio acadêmico. Nesse sentido, um importante passo para o preenchimento desta lacuna vem sendo dado pelo CT 702 - Comitê IBRACON/ ALCONPAT, que busca apresentar procedimentos para ensaios, inspeção e avaliação da durabilidade de estruturas de concreto, em uma valiosa iniciativa para a produção de referenciais normativos brasileiros sobre o tema.

\section{AGRADECIMENTOS}

O autor Gustavo B. Wally agradece à Coordenação de Aperfeiçoamento de Pessoal de Nível Superior - Brasil (CAPES) pelo auxílio financeiro concedido através de bolsa de doutorado.

\section{DREFRÊNCIAS BIBLIOGRÁFICAS}

[1] ALEXANDER, M.; THOMAS, M. Service life prediction and performance testing - Current developments and practical applications. Cement and Concrete Research, 78-A, p. 155-164, 2015.

[2] ASSOCIAÇÃO BRASILEIRA DE NORMAS TÉCNICAS. ABNT NBR 6118: Projeto de estruturas de concreto - Procedimento. Rio de Janeiro: ABNT, 2014

[3] BAROGHEL-BOUNY, V. et al. Easy assessment of durability indicators for service life prediction or quality control of concretes with high volumes of supplementary cementitious materials. Cement and Concrete Composites, 33, 8, p. 832-847, 2011.

[4] BAROGHEL-BOUNY, V.; NGUYEN, T. Q.; DANGLA, P. Assessment and prediction of RC structure service life by means of durability indicators and physical/chemical model. Cement and Concrete Composites, 31, 8, p. 522-534, 2009.

[5] BEUSHAUSEN, H. et al. Principles of the Performance-Bases Approach for Concrete Durability. In: BEUSHAUSEN, H.; LUCO, L. F. (coord.). Performance-Based Specifications and Control of Concrete Durability. New York: Springer, 2016, p. 107-131.

[6] BEUSHAUSEN, H.; TORRENT, R.; ALEXANDER, M. G. Performance-based approaches for concrete durability: State of the art and future research needs. Cement and Concrete Research, 119, p. 11-20, 2019.

[7] EUROPEAN COMMITTEE FOR STANDARDIZATION. EN 206: Concrete Part I: Specification, Performance, Production and Conformity. Brussels: CEN, 2007.

[8] GJØRV, 0. E. Projeto de durabilidade de estruturas de concreto em ambientes de severa agressividade (Ed. Trad.). São Paulo: Oficina de Textos, 2015, 238 p.

[9] SCHWEIZERISCHER INGENIEUR- UND ARCHITEKTENVEREIN. SIA 262: Betonbau. Zürich: SIA, 2013.

[10] TORRENT, R. J. Bridge durability design after EN standards: present and future. Structure and Infrastructure Engineering, 15, 7, p. 886-898, 2018.

[11] TORRENT, R. et al. Prescriptive Durability Specifications. In: BEUSHAUSEN, H.; LUCO, L. F. (coord.). Performance-Based Specifications and Control of Concrete Durability. New York: Springer, 2016, p. 19-49.

[12] WALLY, G. B. Vida útil de concretos produzidos com sílica ativa e metacaulim frente ao ataque por cloretos: Indicadores de durabilidade e análise probabilística. Dissertação de Mestrado, Programa de Pós-Graduação em Engenharia Oceânica, Universidade Federal do Rio Grande, Rio Grande, 2019. 